\title{
Approximate solutions for MHD squeezing fluid flow by a novel method
}

Mustafa Inc ${ }^{1}$ and Ali Akgül ${ }^{2,3 *}$

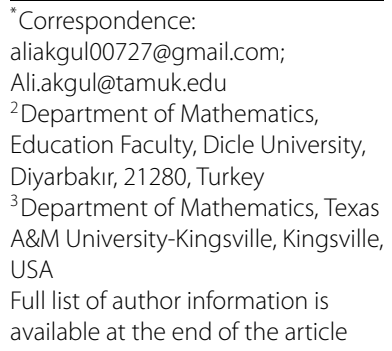

${ }^{3}$ Department of Mathematics, Texas A\&M University-Kingsville, Kingsville, USA

Full list of author information is available at the end of the article

\begin{abstract}
In this paper, a steady axisymmetric MHD flow of two-dimensional incompressible fluids has been investigated. The reproducing kernel Hilbert space method (RKHSM) has been implemented to obtain a solution of the reduced fourth-order nonlinear boundary value problem. Numerical results have been compared with the results obtained by the Runge-Kutta method (RK-4) and optimal homotopy asymptotic method (OHAM).
\end{abstract}

MSC: 46E22; 35A24

Keywords: reproducing kernel method; series solutions; squeezing fluid flow; magnetohydrodynamics; reproducing kernel space

\section{Introduction}

Squeezing flows have many applications in food industry, principally in chemical engineering [1-4]. Some practical examples of squeezing flow include polymer processing, compression and injection molding. Grimm [5] studied numerically the thin Newtonian liquids films being squeezed between two plates. Squeezing flow coupled with magnetic field is widely applied to bearing with liquid-metal lubrication [2, 6-8].

In this paper, we use RKHSM to study the squeezing MHD fluid flow between two infinite planar plates. This problem has been solved by RKHSM and for comparison it has been compared with the OHAM and numerically with the RK-4 by using Maple 16.

The RKHSM, which accurately computes the series solution, is of great interest to applied sciences. The method provides the solution in a rapidly convergent series with components that can be elegantly computed. The efficiency of the method was used by many authors to investigate several scientific applications. Geng and Cui [9] and Zhou et al. [10] applied the RKHSM to handle the second-order boundary value problems. Yao and Cui [11] and Wang et al. [12] investigated a class of singular boundary value problems by this method and the obtained results were good. Wang and Chao [13], Li and Cui [14], Zhou and Cui [15] independently employed the RKSHSM to variable-coefficient partial differential equations. Du and Cui [16] investigated the approximate solution of the forced Duffing equation with integral boundary conditions by combining the homotopy perturbation method and the RKM. Lv and Cui [17] presented a new algorithm to solve linear fifth-order boundary value problems. Cui and $\mathrm{Du}$ [18] obtained the representation of the exact solution for the nonlinear Volterra-Fredholm integral equations by using the RKHSM. Wu and $\mathrm{Li}$ [19] applied iterative RKHSM to obtain the analytical approximate solution of a non-

\section{Springer}

○2014 Inc and Akgül; licensee Springer. This is an Open Access article distributed under the terms of the Creative Commons Attribution License (http://creativecommons.org/licenses/by/2.0), which permits unrestricted use, distribution, and reproduction in any medium, provided the original work is properly cited. 
linear oscillator with discontinuities. For more details about RKHSM and the modified forms and its effectiveness, see [9-37] and the references therein.

The paper is organized as follows. We give the problem formulation in Section 2. Section 3 introduces several reproducing kernel spaces. A bounded linear operator is presented in Section 4. In Section 5, we provide the main results, the exact and approximate solutions. An iterative method is developed for the kind of problems in the reproducing kernel space. We prove that the approximate solution converges to the exact solution uniformly. Some numerical experiments are illustrated in Section 6. There are some conclusions in the last section.

\section{Problem formulation}

Consider a squeezing flow of an incompressible Newtonian fluid in the presence of a magnetic field of a constant density $\rho$ and viscosity $\mu$ squeezed between two large planar parallel plates separated by a small distance $2 H$ and the plates approaching each other with a low constant velocity $V$, as illustrated in Figure 1, and the flow can be assumed to quasisteady $[1-3,39]$. The Navier-Stokes equations $[3,4]$ governing such flow in the presence of magnetic field, when inertial terms are retained in the flow, are given as [38]

$$
\nabla V \cdot u=0
$$

and

$$
\rho\left[\frac{\partial u}{\partial t}+(u \cdot \nabla) u\right]=\nabla \cdot T+J \times B+\rho f,
$$

where $u$ is the velocity vector, $\nabla$ denotes the material time derivative, $T$ is the Cauchy stress tensor,

$$
T=-p I+\mu A_{1}
$$

and

$$
A_{1}=\nabla u+u^{T},
$$

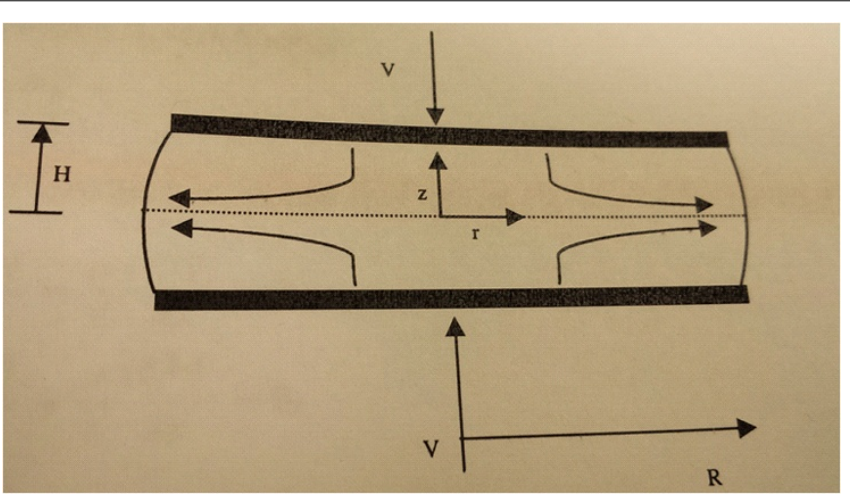

Figure 1 A steady squeezing axisymmetric fluid flow between two parallel plates [38]. 
$J$ is the electric current density, $B$ is the total magnetic field and

$$
B=B_{0}+b,
$$

$B_{0}$ represents the imposed magnetic field and $b$ denotes the induced magnetic field. In the absence of displacement currents, the modified Ohm law and Maxwell's equations (see [40] and the references therein) are given by [38]

$$
J=\sigma[E+u \times B]
$$

and

$$
\operatorname{div} B=0, \quad \nabla \times B=\mu_{m} J, \quad \operatorname{curl} E=\frac{\partial B}{\partial t},
$$

in which $\sigma$ is the electrical conductivity, $E$ is the electric field and $\mu_{m}$ is the magnetic permeability.

The following assumptions are needed [38].

(a) The density $\rho$, magnetic permeability $\mu_{m}$ and electric field conductivity $\sigma$ are assumed to be constant throughout the flow field region.

(b) The electrical conductivity $\sigma$ of the fluid is considered to be finite.

(c) Total magnetic field $B$ is perpendicular to the velocity field $V$ and the induced magnetic field $b$ is negligible compared with the applied magnetic field $B_{0}$ so that the magnetic Reynolds number is small (see [40] and the references therein).

(d) We assume a situation where no energy is added or extracted from the fluid by the electric field, which implies that there is no electric field present in the fluid flow region.

Under these assumptions, the magnetohydrodynamic force involved in Eq. (2.2) can be put into the form

$$
J \times B=-\sigma B_{0}^{2} u .
$$

An axisymmetric flow in cylindrical coordinates $r, \theta, z$ with $z$-axis perpendicular to plates and $z= \pm H$ at the plates. Since we have axial symmetry, $u$ is represented by

$$
u=\left(u_{r}(r, z), 0, u_{z}(r, z)\right),
$$

when body forces are negligible, Navier-Stokes Eqs. (2.1)-(2.2) in cylindrical coordinates, where there is no tangential velocity $\left(u_{\theta}=0\right)$, are given as [38]

$$
\rho\left(u_{r} \frac{\partial u_{r}}{\partial r}+u_{z} \frac{\partial u_{r}}{\partial z}\right)=-\frac{\partial p}{\partial r}+\left(\frac{\partial^{2} u_{r}}{\partial r^{2}}+\frac{1}{r} \frac{\partial u_{r}}{\partial r}-\frac{u_{r}}{r^{2}}+\frac{\partial^{2} u_{r}}{\partial z^{2}}\right)+\sigma B_{0}^{2} u
$$

and

$$
\rho\left(u_{z} \frac{\partial u_{z}}{\partial r}+u_{z} \frac{\partial u_{z}}{\partial z}\right)=-\frac{\partial p}{\partial r}+\left(\frac{\partial^{2} u_{z}}{\partial r^{2}}+\frac{1}{r} \frac{\partial u_{z}}{\partial r}+\frac{\partial^{2} u_{z}}{\partial z^{2}}\right),
$$


where $p$ is the pressure, and the equation of continuity is given by [38]

$$
\frac{1}{r} \frac{\partial}{\partial r}\left(r u_{r}\right)+\frac{\partial u_{z}}{\partial z}=0
$$

The boundary conditions require

$$
\begin{array}{lll}
u_{r}=0, & u_{z}=-V & \text { at } z=H, \\
\frac{\partial u_{r}}{\partial z}=0, & u_{z}=0 & \text { at } z=0 .
\end{array}
$$

Let us introduce the axisymmetric Stokes stream function $\Psi$ as

$$
u_{r}=\frac{1}{r} \frac{\partial \Psi}{\partial z}, \quad u_{z}=-\frac{1}{r} \frac{\partial \Psi}{\partial r} .
$$

The continuity equation is satisfied using Eq. (2.10). Substituting Eqs. (2.3)-(2.5) and Eq. (2.10) into Eqs. (2.7)-(2.8), we obtain

$$
-\frac{\rho}{r^{2}} \frac{\partial \Psi}{\partial r} E^{2} \Psi=-\frac{\partial p}{\partial r}+\frac{\mu}{r} \frac{\partial E^{2} \Psi}{\partial z}-\frac{\sigma B_{0}^{2}}{r} \frac{\partial \Psi}{\partial z}
$$

and

$$
-\frac{\rho}{r^{2}} \frac{\partial \Psi}{\partial z} E^{2} \Psi=-\frac{\partial p}{\partial z}+\frac{\mu}{r} \frac{\partial E^{2} \Psi}{\partial r} .
$$

Eliminating the pressure from Eqs. (2.11) and (2.12) by the integrability condition, we get the compatibility equation as [38]

$$
-\rho\left[\frac{\partial\left(\Psi, \frac{E^{2} \Psi}{r^{2}}\right)}{\partial(r, z)}\right]=\frac{\mu}{r} E^{2} \Psi-\frac{\sigma B_{0}^{2}}{r} \frac{\partial^{2 \Psi}}{\partial z^{2}},
$$

where

$$
E^{2}=\frac{\partial^{2}}{\partial r^{2}}-\frac{1}{r} \frac{\partial}{\partial r}+\frac{\partial^{2}}{\partial z^{2}} .
$$

The stream function can be expressed as $[1,3]$

$$
\Psi(r, z)=r^{2} F(z) .
$$

In view of Eq. (2.14), the compatibility equation (2.13) and the boundary conditions (2.9) take the form

$$
F^{(i v)}(z)-\frac{\sigma B_{0}^{2}}{r} F^{\prime \prime}(z)+2 \frac{\rho}{\mu} F(z) F^{\prime \prime \prime}(z)=0,
$$

subject to

$$
\begin{array}{ll}
F(0)=0, & F^{\prime \prime}(0)=0, \\
F(H)=\frac{V}{2}, & F^{\prime}(H)=0 .
\end{array}
$$


Non-dimensional parameters are given as [38]

$$
F^{*}=2 \frac{F}{V}, \quad z^{*}=\frac{z}{H}, \quad \operatorname{Re}=\frac{\rho H V}{\mu}, \quad m=B_{0} H \sqrt{\frac{\sigma}{\mu}} .
$$

For simplicity omitting the $*$, the boundary value problem (2.15)-(2.16) becomes [38]

$$
F^{(i v)}(z)-m^{2} F^{\prime \prime}(z)+\operatorname{Re} F(z) F^{\prime \prime \prime}(z)=0
$$

with the boundary conditions

$$
\begin{array}{ll}
F(0)=0, & F^{\prime \prime}(0)=0, \\
F(1)=1, & F^{\prime}(1)=0,
\end{array}
$$

where Re is the Reynolds number and $m$ is the Hartmann number.

\section{Reproducing kernel spaces}

In this section, we define some useful reproducing kernel spaces.

Definition 3.1 (Reproducing kernel) Let $E$ be a nonempty abstract set. A function $K$ : $E \times E \rightarrow C$ is a reproducing kernel of the Hilbert space $H$ if and only if

$$
\left\{\begin{array}{l}
\forall t \in E, \quad K(\cdot, t) \in H \\
\forall t \in E, \forall \varphi \in H, \quad\langle\varphi(\cdot), K(\cdot, t)\rangle=\varphi(t) .
\end{array}\right.
$$

The last condition is called 'the reproducing property': the value of the function $\varphi$ at the point $t$ is reproduced by the inner product of $\varphi$ with $K(\cdot, t)$.

Definition 3.2 We define the space $W_{2}^{5}[0,1]$ by

$$
W_{2}^{5}[0,1]=\left\{\begin{array}{l}
u \mid u, u^{\prime}, u^{\prime \prime}, u^{\prime \prime \prime}, u^{(4)} \text { are absolutely continuous in }[0,1] \\
u^{(5)} \in L^{2}[0,1], x \in[0,1], u(0)=u(1)=u^{\prime}(1)=u^{\prime \prime}(0)=0
\end{array}\right\}
$$

The fifth derivative of $u$ exists almost everywhere since $u^{(4)}$ is absolutely continuous. The inner product and the norm in $W_{2}^{5}[0,1]$ are defined respectively by

$$
\langle u, v\rangle_{W_{2}^{5}}=\sum_{i=0}^{4} u^{(i)}(0) v^{(i)}(0)+\int_{0}^{1} u^{(5)}(x) v^{(5)}(x) d x, \quad u, v \in W_{2}^{5}[0,1]
$$

and

$$
\|u\|_{W_{2}^{5}}=\sqrt{\langle u, u\rangle_{W_{2}^{5}}}, \quad u \in W_{2}^{5}[0,1]
$$

The space $W_{2}^{5}[0,1]$ is a reproducing kernel space, i.e., for each fixed $y \in[0,1]$ and any $u \in W_{2}^{5}[0,1]$, there exists a function $R_{y}$ such that

$$
u=\left\langle u, R_{y}\right\rangle_{W_{2}^{5}} \text {. }
$$


Definition 3.3 We define the space $W_{2}^{4}[0,1]$ by

$$
W_{2}^{4}[0,1]=\left\{\begin{array}{l}
u \mid u, u^{\prime}, u^{\prime \prime}, u^{\prime \prime \prime} \text { are absolutely continuous in }[0,1], \\
u^{(4)} \in L^{2}[0,1], x \in[0,1]
\end{array}\right\} .
$$

The fourth derivative of $u$ exists almost everywhere since $u^{(3)}$ is absolutely continuous. The inner product and the norm in $W_{2}^{4}[0,1]$ are defined respectively by

$$
\langle u, v\rangle_{W_{2}^{4}}=\sum_{i=0}^{3} u^{(i)}(0) v^{(i)}(0)+\int_{0}^{1} u^{(4)}(x) v^{(4)}(x) d x, \quad u, v \in W_{2}^{4}[0,1]
$$

and

$$
\|u\|_{W_{2}^{4}}=\sqrt{\langle u, u\rangle_{W_{2}^{4}}}, \quad u \in W_{2}^{4}[0,1] .
$$

The space $W_{2}^{4}[0,1]$ is a reproducing kernel space, i.e., for each fixed $y \in[0,1]$ and any $u \in W_{2}^{4}[0,1]$, there exists a function $r_{y}$ such that

$$
u=\left\langle u, r_{y}\right\rangle_{W_{2}^{4}}
$$

Theorem 3.1 The space $W_{2}^{5}[0,1]$ is a reproducing kernel Hilbert space whose reproducing kernel function is given by

$$
R_{y}(x)= \begin{cases}\sum_{i=1}^{10} c_{i}(y) x^{i-1}, & x \leq y \\ \sum_{i=1}^{10} d_{i}(y) x^{i-1}, & x>y\end{cases}
$$

where $c_{i}(y)$ and $d_{i}(y)$ can be obtained easily by using Maple 16 and the proof of Theorem 3.1 is given in Appendix.

Remark 3.1 The reproducing kernel function $r_{y}$ of $W_{2}^{4}[0,1]$ is given as

$$
r_{y}(x)= \begin{cases}1+x y+\frac{1}{4} y^{2} x^{2}+\frac{1}{36} y^{3} x^{3}+\frac{1}{144} y^{3} x^{4}-\frac{1}{240} y^{2} x^{5}+\frac{1}{720} y x^{6}-\frac{1}{5,040} x^{7}, & x \leq y, \\ 1+y x+\frac{1}{4} y^{2} x^{2}+\frac{1}{36} y^{3} x^{3}+\frac{1}{144} x^{3} y^{4}-\frac{1}{240} x^{2} y^{5}+\frac{1}{720} x y^{6}-\frac{1}{5,040} y^{7}, & x>y .\end{cases}
$$

This can be proved easily as the proof of Theorem 3.1.

\section{Bounded linear operator in $W_{2}^{5}[0,1]$}

In this section, the solution of Eq. (2.17) is given in the reproducing kernel space $W_{2}^{5}[0,1]$.

On defining the linear operator $L: W_{2}^{5}[0,1] \rightarrow W_{2}^{4}[0,1]$ as

$$
L u=u^{(4)}(x)+\operatorname{Re} \frac{e^{x}}{e}\left(x^{3}-4 x^{2}+4 x\right) u^{(3)}(x)-m^{2} u^{\prime \prime}(x)+\operatorname{Re} \frac{e^{x}}{e}\left(x^{3}+5 x^{2}-2 x-6\right) u(x) .
$$

Model problem (2.17)-(2.18) changes the following problem:

$$
\left\{\begin{array}{l}
L u=M\left(x, u, u^{(3)}\right), \quad x \in[0,1] \\
u(0)=0, \quad u(1)=0, \quad u^{\prime}(1)=0, \quad u^{\prime \prime}(0)=0
\end{array}\right.
$$


where

$$
F(x)=u(x)+\frac{e^{x}}{e}\left(x^{3}-4 x^{2}+4 x\right)
$$

and

$$
\begin{aligned}
M\left(x, u, u^{(3)}\right)= & -\operatorname{Re} u^{(3)}(x) u(x)-\operatorname{Re}\left(\frac{e^{x}}{e}\right)^{2}\left(x^{3}-4 x^{2}+4 x\right)\left(x^{3}+5 x^{2}-2 x-6\right) \\
& -\frac{e^{x}}{e}\left(x^{3}+8 x^{2}+8 x-2\right)+m^{2} \frac{e^{x}}{e}\left(x^{3}+2 x^{2}-6 x\right) .
\end{aligned}
$$

Theorem 4.1 The operator $L$ defined by (4.1) is a bounded linear operator.

Proof We only need to prove

$$
\|L u\|_{W_{2}^{4}}^{2} \leq P\|L u\|_{W_{2}^{5}}^{2},
$$

where $P$ is a positive constant. By Definition 3.3, we have

$$
\|u\|_{W_{2}^{4}}^{2}=\langle u, u\rangle_{W_{2}^{4}}=\sum_{i=0}^{3}\left[u^{(i)}(0)\right]^{2}+\int_{0}^{1}\left[u^{(4)}(x)\right]^{2} d x, \quad u \in W_{2}^{4}[0,1],
$$

and

$$
\begin{aligned}
\|L u\|_{W_{2}^{4}}^{2}=\langle L u, L u\rangle_{W_{2}^{4}}= & {[(L u)(0)]^{2}+\left[(L u)^{\prime}(0)\right]^{2}+\left[(L u)^{\prime \prime}(0)\right]^{2} } \\
& +\left[(L u)^{(3)}(0)\right]^{2}+\int_{0}^{1}\left[(L u)^{(4)}(x)\right]^{2} d x .
\end{aligned}
$$

By the reproducing property, we have

$$
u(x)=\left\langle u, R_{x}\right\rangle_{W_{2}^{5}},
$$

and

$$
\begin{aligned}
& (L u)(x)=\left\langle u,\left(L R_{x}\right)\right\rangle_{W_{2}^{5}}, \quad(L u)^{\prime}(x)=\left\langle u,\left(L R_{x}\right)^{\prime}\right\rangle_{W_{2}^{5}}, \\
& (L u)^{\prime \prime}(x)=\left\langle u,\left(L R_{x}\right)^{\prime \prime}\right\rangle_{W_{2}^{5}}, \quad(L u)^{(3)}(x)=\left\langle u,\left(L R_{x}\right)^{(3)}\right\rangle_{W_{2}^{5}}, \\
& (L u)^{(4)}(x)=\left\langle u,\left(L R_{x}\right)^{(4)}\right\rangle_{W_{2}^{5} .}
\end{aligned}
$$

Therefore, by the Cauchy-Schwarz inequality, we get

$$
\begin{aligned}
& |(L u)(x)| \leq\|u\|_{W_{2}^{5}}\left\|L R_{x}\right\|_{W_{2}^{5}}=a_{1}\|u\|_{W_{2}^{5}} \quad \text { (where } a_{1}>0 \text { is a positive constant), } \\
& \left|(L u)^{\prime}(x)\right| \leq\|u\|_{W_{2}^{5}}\left\|\left(L R_{x}\right)^{\prime}\right\|_{W_{2}^{5}}=a_{2}\|u\|_{W_{2}^{5}} \quad \text { (where } a_{2}>0 \text { is a positive constant), } \\
& \left|(L u)^{\prime \prime}(x)\right| \leq\|u\|_{W_{2}^{5}}\left\|\left(L R_{x}\right)^{\prime \prime}\right\|_{W_{2}^{5}}=a_{3}\|u\|_{W_{2}^{5}} \quad \text { (where } a_{3}>0 \text { is a positive constant), } \\
& \left|(L u)^{(3)}(x)\right| \leq\|u\|_{W_{2}^{5}}\left\|\left(L R_{x}\right)^{(3)}\right\|_{W_{2}^{5}}=a_{4}\|u\|_{W_{2}^{5}} \quad \text { (where } a_{4}>0 \text { is a positive constant). }
\end{aligned}
$$


Thus

$$
[(L u)(0)]^{2}+\left[(L u)^{\prime}(0)\right]^{2}+\left[(L u)^{\prime \prime}(0)\right]^{2}+\left[(L u)^{(3)}(0)\right]^{2} \leq\left(a_{1}^{2}+a_{2}^{2}+a_{3}^{2}+a_{4}^{2}\right)\|u\|_{W_{2}^{5}}^{2}
$$

Since

$$
(L u)^{(4)}=\left\langle u,\left(L R_{x}\right)^{(4)}\right\rangle_{W_{2}^{5}},
$$

then

$$
\left.\left|(L u)^{(4)}\right| \leq\|u\|_{W_{2}^{5}}\left\|\left(L R_{x}\right)^{(4)}\right\|_{W_{2}^{5}}=a_{5}\|u\|_{W_{2}^{4}} \quad \text { (where } a_{5}>0 \text { is a positive constant }\right) .
$$

Therefore, we have

$$
\left[(L u)^{(4)}\right]^{2} \leq a_{5}^{2}\|u\|_{W_{2}^{5}}^{2}
$$

and

$$
\int_{0}^{1}\left[(L u)^{(4)}(x)\right]^{2} d x \leq a_{5}^{2}\|u\|_{W_{2}^{5}}^{2}
$$

that is,

$$
\begin{aligned}
\|L u\|_{W_{2}^{4}}^{2} & =[(L u)(0)]^{2}+\left[(L u)^{\prime}(0)\right]^{2}+\left[(L u)^{\prime \prime}(0)\right]^{2}+\left[(L u)^{(3)}(0)\right]^{2}+\int_{0}^{1}\left[(L u)^{(4)}(x)\right]^{2} d x \\
& \leq\left(a_{1}^{2}+a_{2}^{2}+a_{3}^{2}+a_{4}^{2}+a_{5}^{2}\right)\|u\|_{W_{2}^{5}}^{2}=P\|u\|_{W_{2}^{4}}^{2},
\end{aligned}
$$

where $P=\left(a_{1}^{2}+a_{2}^{2}+a_{3}^{2}+a_{4}^{2}+a_{5}^{2}\right)>0$ is a positive constant. This completes the proof.

\section{Analysis of the solution of (2.17)-(2.18)}

Let $\left\{x_{i}\right\}_{i=1}^{\infty}$ be any dense set in $[0,1]$ and $\Psi_{x}(y)=L^{*} r_{x}(y)$, where $L^{*}$ is the adjoint operator of $L$ and $r_{x}$ is given by Remark 3.1. Furthermore

$$
\Psi_{i}(x) \stackrel{\text { def }}{=} \Psi_{x_{i}}(x)=L^{*} r_{x_{i}}(x)
$$

Lemma 5.1 $\left\{\Psi_{i}(x)\right\}_{i=1}^{\infty}$ is a complete system of $W_{2}^{5}[0,1]$.

Proof For $u \in W_{2}^{5}[0,1]$, let

$$
\left\langle u, \Psi_{i}\right\rangle=0 \quad(i=1,2, \ldots)
$$

that is,

$$
\left\langle u, L^{*} r_{x_{i}}\right\rangle=(L u)\left(x_{i}\right)=0
$$

Note that $\left\{x_{i}\right\}_{i=1}^{\infty}$ is the dense set in $[0,1]$. Therefore $(L u)(x)=0$. Assume that (4.1) has a unique solution. Then $L$ is one-to-one on $W_{2}^{5}[0,1]$ and thus $u(x)=0$. This completes the proof. 
Lemma 5.2 The following formula holds:

$$
\Psi_{i}(x)=\left(L_{\eta} R_{x}(\eta)\right)\left(x_{i}\right)
$$

where the subscript $\eta$ of the operator $L_{\eta}$ indicates that the operator $L$ applies to a function of $\eta$.

Proof

$$
\begin{aligned}
\Psi_{i}(x) & =\left\langle\Psi_{i}(\xi), R_{x}(\xi)\right\rangle_{W_{2}^{5}[0,1]} \\
& =\left\langle L^{*} r_{x_{i}}(\xi), R_{x}(\xi)\right\rangle_{W_{2}^{5}[0,1]} \\
& =\left\langle\left(r_{x_{i}}\right)(\xi),\left(L_{\eta} R_{x}(\eta)\right)(\xi)\right\rangle_{W_{2}^{4}[0,1]} \\
& =\left(L_{\eta} R_{x}(\eta)\right)\left(x_{i}\right) .
\end{aligned}
$$

This completes the proof.

Remark 5.1 The orthonormal system $\left\{\bar{\Psi}_{i}(x)\right\}_{i=1}^{\infty}$ of $W_{2}^{5}[0,1]$ can be derived from the Gram-Schmidt orthogonalization process of $\left\{\Psi_{i}(x)\right\}_{i=1}^{\infty}$ as

$$
\bar{\Psi}_{i}(x)=\sum_{k=1}^{i} \beta_{i k} \Psi_{k}(x) \quad\left(\beta_{i i}>0, i=1,2, \ldots\right),
$$

where $\beta_{i k}$ are orthogonal coefficients.

In the following, we give the representation of the exact solution of Eq. (2.17) in the reproducing kernel space $W_{2}^{5}[0,1]$.

Theorem 5.1 If $u$ is the exact solution of (4.1), then

$$
u=\sum_{i=1}^{\infty} \sum_{k=1}^{i} \beta_{i k} M\left(x_{k}, u\left(x_{k}\right), u^{(3)}\left(x_{k}\right)\right) \bar{\Psi}_{i}(x)
$$

where $\left\{x_{i}\right\}_{i=1}^{\infty}$ is a dense set in $[0,1]$.

Proof From (5.1) and the uniqueness of solution of (4.1), we have

$$
\begin{aligned}
u & =\sum_{i=1}^{\infty}\left\langle u, \bar{\Psi}_{i}\right\rangle_{W_{2}^{5}} \bar{\Psi}_{i}=\sum_{i=1}^{\infty} \sum_{k=1}^{i} \beta_{i k}\left\langle u, L^{*} r_{x_{k}}\right\rangle_{W_{2}^{5}} \bar{\Psi}_{i} \\
& =\sum_{i=1}^{\infty} \sum_{k=1}^{i} \beta_{i k}\left\langle L u, r_{x_{k}}\right\rangle_{W_{2}^{4}} \bar{\Psi}_{i}=\sum_{i=1}^{\infty} \sum_{k=1}^{i} \beta_{i k}\left\langle M\left(x, u, u^{(3)}\right), r_{x_{k}}\right\rangle_{W_{2}^{4}} \bar{\Psi}_{i} \\
& =\sum_{i=1}^{\infty} \sum_{k=1}^{i} \beta_{i k} M\left(x_{k}, u\left(x_{k}\right), u^{(3)}\left(x_{k}\right)\right) \bar{\Psi}_{i}(x) .
\end{aligned}
$$

This completes the proof. 
Now the approximate solution $u_{n}$ can be obtained by truncating the $n$-term of the exact solution $u$ as

$$
u_{n}=\sum_{i=1}^{n} \sum_{k=1}^{i} \beta_{i k} M\left(x_{k}, u\left(x_{k}\right), u^{(3)}\left(x_{k}\right)\right) \bar{\Psi}_{i}(x) .
$$

Lemma 5.3 ([30]) Assume that $u$ is the solution of (4.1) and $r_{n}$ is the error between the approximate solution $u_{n}$ and the exact solution $u$. Then the error sequence $r_{n}$ is monotone decreasing in the sense of $\|\cdot\|_{W_{2}^{5}}$ and $\left\|r_{n}(x)\right\|_{W_{2}^{5}} \rightarrow 0$.

\section{Numerical results}

In this section, comparisons of results are made through different Reynolds numbers Re and magnetic field effect $m$. All computations are performed by Maple 16. Figure 5.7 shows comparisons of $F(z)$ for a fixed Reynolds number with increasing magnetic field effect $m=1,3,8,20$. From this figure, the velocity decreases due to an increase in $m$. Figure 5.8 shows comparisons of $F(z)$ for a fixed magnetic field $m=1$ with increasing Reynolds numbers $\operatorname{Re}=1,4,10$. It is observed that much increase in Reynolds numbers affects the results. The RKHSM does not require discretization of the variables, i.e., time and space, it is not affected by computation round of errors and one is not faced with necessity of large computer memory and time. The accuracy of the RKHSM for the MHD squeezing fluid flow is controllable and absolute errors are small with present choice of $x$ (see Tables 1-6 and Figures 2-7). The numerical results we obtained justify the advantage of this methodology. Generally it is not possible to find the exact solution of these problems.

Table 1 Numerical results at $m=1$ and $R e=1$

\begin{tabular}{lllllll}
\hline $\boldsymbol{x}$ & OHAM & $\begin{array}{l}\text { Numerical solution } \\
\text { (RK-4) }\end{array}$ & $\begin{array}{l}\text { Approximate solution } \\
\text { RKHSM }\end{array}$ & $\begin{array}{l}\text { Absolute } \\
\text { error }\end{array}$ & $\begin{array}{l}\text { Relative } \\
\text { error }\end{array}$ & \begin{tabular}{l} 
Time (s) \\
\hline 0.1
\end{tabular} \\
\hline 0.150265 & 0.150294 & 0.15029400074386619072 & $7.43 \times 10^{-10}$ & $4.94 \times 10^{-9}$ & 2.948 \\
0.2 & 0.297424 & 0.297481 & 0.29748099943286204844 & $5.67 \times 10^{-10}$ & $1.9 \times 10^{-9}$ & 2.980 \\
0.3 & 0.438387 & 0.438467 & 0.43846699936146542481 & $6.38 \times 10^{-10}$ & $1.45 \times 10^{-9}$ & 2.870 \\
0.4 & 0.570093 & 0.570189 & 0.57018899983086605298 & $1.69 \times 10^{-10}$ & $2.96 \times 10^{-10}$ & 2.792 \\
0.5 & 0.68952 & 0.689624 & 0.68962399932753349664 & $6.72 \times 10^{-10}$ & $9.75 \times 10^{-10}$ & 2.824 \\
0.6 & 0.793695 & 0.793796 & 0.79379600052975674440 & $5.29 \times 10^{-10}$ & $6.67 \times 10^{-10}$ & 2.902 \\
0.7 & 0.879695 & 0.879779 & 0.87977900034152532706 & $3.41 \times 10^{-10}$ & $3.88 \times 10^{-10}$ & 2.964 \\
0.8 & 0.944641 & 0.944696 & 0.94469600021478585921 & $2.14 \times 10^{-10}$ & $2.27 \times 10^{-10}$ & 2.808 \\
0.9 & 0.985687 & 0.985707 & 0.98570699945336089741 & $5.46 \times 10^{-10}$ & $5.54 \times 10^{-10}$ & 2.761 \\
1.0 & 1.0 & 1.0 & 1.0 & 0.0 & 0.0 & 2.902 \\
\hline
\end{tabular}

Table 2 Numerical results at $m=3$ and $\operatorname{Re}=1$

\begin{tabular}{lllllll}
\hline $\boldsymbol{x}$ & OHAM & $\begin{array}{l}\text { Numerical solution } \\
\text { (RK-4) }\end{array}$ & $\begin{array}{l}\text { Approximate solution } \\
\text { RKHSM }\end{array}$ & $\begin{array}{l}\text { Absolute } \\
\text { error }\end{array}$ & $\begin{array}{l}\text { Relative } \\
\text { error }\end{array}$ & \begin{tabular}{l} 
Time (s) \\
\hline 0.1
\end{tabular} \\
\hline 0.13709 & 0.137044 & 0.13704399924397146430 & $7.56 \times 10^{-10}$ & $5.51 \times 10^{-9}$ & 3.261 \\
0.2 & 0.272583 & 0.272494 & 0.27249400041809657591 & $4.18 \times 10^{-10}$ & $1.53 \times 10^{-9}$ & 3.542 \\
0.3 & 0.404759 & 0.404637 & 0.40463699937791012358 & $6.22 \times 10^{-10}$ & $1.53 \times 10^{-9}$ & 2.949 \\
0.4 & 0.531649 & 0.531508 & 0.53150799980699743080 & $1.93 \times 10^{-10}$ & $3.63 \times 10^{-10}$ & 3.541 \\
0.5 & 0.650894 & 0.650756 & 0.65075599905912100256 & $9.4 \times 10^{-10}$ & $1.44 \times 10^{-9}$ & 3.089 \\
0.6 & 0.759591 & 0.759478 & 0.75947799979255971384 & $2.07 \times 10^{-10}$ & $2.73 \times 10^{-10}$ & 2.996 \\
0.7 & 0.854106 & 0.854035 & 0.85403499924057783299 & $7.59 \times 10^{-10}$ & $8.89 \times 10^{-10}$ & 3.026 \\
0.8 & 0.929845 & 0.929817 & 0.92981700082221438640 & $8.22 \times 10^{-10}$ & $8.84 \times 10^{-10}$ & 7.582 \\
0.9 & 0.980966 & 0.980963 & 0.98096299961587653980 & $3.84 \times 10^{-10}$ & $3.91 \times 10^{-10}$ & 3.291 \\
1.0 & 1.0 & 1.0 & 1.0 & 0.0 & 0.0 & 2.902 \\
\hline
\end{tabular}


Table 3 Numerical results at $m=8$ and $R e=1$

\begin{tabular}{lllllll}
\hline $\boldsymbol{x}$ & OHAM & $\begin{array}{l}\text { Numerical solution } \\
\text { (RK-4) }\end{array}$ & $\begin{array}{l}\text { Approximate solution } \\
\text { RKHSM }\end{array}$ & $\begin{array}{l}\text { Absolute } \\
\text { error }\end{array}$ & $\begin{array}{l}\text { Relative } \\
\text { error }\end{array}$ & Time (s) \\
\hline 0.1 & 0.11507 & 0.114976 & 0.11497599095960418967 & $9.04 \times 10^{-9}$ & $7.86 \times 10^{-8}$ & 4.290 \\
0.2 & 0.230068 & 0.229882 & 0.22988199268533318687 & $7.31 \times 10^{-9}$ & $3.18 \times 10^{-8}$ & 4.134 \\
0.3 & 0.344866 & 0.344604 & 0.34460400584434350472 & $5.84 \times 10^{-9}$ & $1.69 \times 10^{-8}$ & 4.477 \\
0.4 & 0.459205 & 0.458904 & 0.45890399132822355411 & $8.67 \times 10^{-9}$ & $1.88 \times 10^{-8}$ & 4.275 \\
0.5 & 0.572545 & 0.572276 & 0.5722759999680104400 & $3.19 \times 10^{-11}$ & $5.58 \times 10^{-11}$ & 3.931 \\
0.6 & 0.683769 & 0.683628 & 0.68362799155831029523 & $8.44 \times 10^{-9}$ & $1.23 \times 10^{-8}$ & 4.556 \\
0.7 & 0.790543 & 0.790607 & 0.79060700783664672119 & $7.83 \times 10^{-9}$ & $9.91 \times 10^{-9}$ & 4.461 \\
0.8 & 0.887936 & 0.888173 & 0.88817300466724146312 & $4.66 \times 10^{-9}$ & $5.25 \times 10^{-9}$ & 3.885 \\
0.9 & 0.965381 & 0.965578 & 0.96557800220185786369 & $2.2 \times 10^{-9}$ & $2.28 \times 10^{-9}$ & 5.007 \\
1.0 & 1.0 & 1.0 & 1.0 & 0.0 & 0.0 & 2.902 \\
\hline
\end{tabular}

Table 4 Numerical results at $m=20$ and $R e=1$

\begin{tabular}{lllllll}
\hline $\boldsymbol{x}$ & OHAM & $\begin{array}{l}\text { Numerical solution } \\
\text { (RK-4) }\end{array}$ & $\begin{array}{l}\text { Approximate solution } \\
\text { RKHSM }\end{array}$ & $\begin{array}{l}\text { Absolute } \\
\text { error }\end{array}$ & $\begin{array}{l}\text { Relative } \\
\text { error }\end{array}$ & Time (s) \\
\hline 0.1 & 0.105312 & 0.105391 & 0.10539098947593257979 & $1.05 \times 10^{-8}$ & $9.98 \times 10^{-8}$ & 4.134 \\
0.2 & 0.210625 & 0.210782 & 0.2107819933190829 & $6.68 \times 10^{-9}$ & $3.16 \times 10^{-8}$ & 5.101 \\
0.3 & 0.315938 & 0.316173 & 0.3161729190893567630 & $8.09 \times 10^{-8}$ & $2.55 \times 10^{-7}$ & 3.010 \\
0.4 & 0.421249 & 0.421563 & 0.4215629919618786430 & $8.03 \times 10^{-9}$ & $1.9 \times 10^{-8}$ & 3.198 \\
0.5 & 0.526551 & 0.526952 & 0.5269519479728988 & $5.2 \times 10^{-8}$ & $9.87 \times 10^{-8}$ & 3.042 \\
0.6 & 0.631824 & 0.632324 & 0.632323981769674315 & $1.82 \times 10^{-8}$ & $2.88 \times 10^{-8}$ & 3.074 \\
0.7 & 0.736971 & 0.737586 & 0.7375860570172070642 & $5.7 \times 10^{-8}$ & $7.73 \times 10^{-8}$ & 3.089 \\
0.8 & 0.841352 & 0.842051 & 0.84205103495023398982 & $3.49 \times 10^{-8}$ & $4.15 \times 10^{-8}$ & 3.073 \\
0.9 & 0.94035 & 0.940861 & 0.94086101815219431313 & $1.81 \times 10^{-8}$ & $1.92 \times 10^{-8}$ & 3.135 \\
1.0 & 1.0 & 1.0 & 1.0 & 0.0 & 0.0 & 2.902 \\
\hline
\end{tabular}

Table 5 Numerical results at $m=1$ and $\operatorname{Re}=4$

\begin{tabular}{lllllll}
\hline $\boldsymbol{x}$ & OHAM & $\begin{array}{l}\text { Numerical solution } \\
\text { (RK-4) }\end{array}$ & $\begin{array}{l}\text { Approximate solution } \\
\text { RKHSM }\end{array}$ & $\begin{array}{l}\text { Absolute } \\
\text { error }\end{array}$ & $\begin{array}{l}\text { Relative } \\
\text { error }\end{array}$ & \begin{tabular}{l} 
Time (s) \\
\hline 0.1
\end{tabular} \\
\hline 0.156218 & 0.158104 & 0.15810400012535311729 & $1.25 \times 10^{-10}$ & $7.92 \times 10^{-10}$ & 5.304 \\
0.2 & 0.308363 & 0.311962 & 0.31196200057873017887 & $5.78 \times 10^{-10}$ & $1.85 \times 10^{-9}$ & 7.332 \\
0.3 & 0.452557 & 0.457539 & 0.45753900003164153289 & $3.16 \times 10^{-11}$ & $6.91 \times 10^{-11}$ & 5.913 \\
0.4 & 0.585287 & 0.591193 & 0.59119300033029000468 & $3.3 \times 10^{-10}$ & $5.58 \times 10^{-10}$ & 6.272 \\
0.5 & 0.703518 & 0.709771 & 0.70977100026331200670 & $2.63 \times 10^{-10}$ & $3.7 \times 10^{-10}$ & 5.757 \\
0.6 & 0.804726 & 0.810642 & 0.81064200064720692438 & $6.47 \times 10^{-10}$ & $7.98 \times 10^{-10}$ & 6.256 \\
0.7 & 0.886838 & 0.891666 & 0.89166599939606220359 & $6.03 \times 10^{-10}$ & $6.03 \times 10^{-10}$ & 6.396 \\
0.8 & 0.948051 & 0.95112 & 0.95112000044608660232 & $4.46 \times 10^{-10}$ & $4.69 \times 10^{-10}$ & 5.101 \\
0.9 & 0.986529 & 0.987612 & 0.98761199979328069240 & $2.06 \times 10^{-10}$ & $2.09 \times 10^{-10}$ & 5.616 \\
1.0 & 1.0 & 1.0 & 1.0 & 0.0 & 0.0 & 2.902 \\
\hline
\end{tabular}

Table 6 Numerical results at $m=1$ and $\operatorname{Re}=10$

\begin{tabular}{lllllll}
\hline $\boldsymbol{x}$ & OHAM & $\begin{array}{l}\text { Numerical solution } \\
\text { (RK-4) }\end{array}$ & $\begin{array}{l}\text { Approximate solution } \\
\text { RKHSM }\end{array}$ & $\begin{array}{l}\text { Absolute } \\
\text { error }\end{array}$ & $\begin{array}{l}\text { Relative } \\
\text { error }\end{array}$ & \begin{tabular}{l} 
Time (s) \\
\hline 0.1
\end{tabular} \\
\hline 0.175911 & 0.167616 & 0.1676160001397322991 & $1.39 \times 10^{-10}$ & $8.33 \times 10^{-10}$ & 5.569 \\
0.2 & 0.344336 & 0.329031 & 0.32903100221406728329 & $2.21 \times 10^{-9}$ & $6.72 \times 10^{-9}$ & 6.365 \\
0.3 & 0.498671 & 0.478907 & 0.47890699791462877619 & $2.08 \times 10^{-9}$ & $4.35 \times 10^{-9}$ & 7.378 \\
0.4 & 0.633941 & 0.613252 & 0.61325199550552162812 & $4.49 \times 10^{-9}$ & $7.32 \times 10^{-9}$ & 7.254 \\
0.5 & 0.747277 & 0.729428 & 0.72942799845508679063 & $1.54 \times 10^{-9}$ & $2.11 \times 10^{-9}$ & 6.271 \\
0.6 & 0.838004 & 0.825843 & 0.82584300690485584332 & $6.9 \times 10^{-9}$ & $8.36 \times 10^{-9}$ & 7.425 \\
0.7 & 0.907244 & 0.901576 & 0.90157600840425340903 & $8.4 \times 10^{-9}$ & $9.32 \times 10^{-9}$ & 6.162 \\
0.8 & 0.956954 & 0.901576 & 0.90157518382496567601 & $8.16 \times 10^{-7}$ & $9.05 \times 10^{-7}$ & 7.410 \\
0.9 & 0.988387 & 0.988978 & 0.98897799997420425356 & $2.57 \times 10^{-11}$ & $2.6 \times 10^{-11}$ & 7.910 \\
1.0 & 1.0 & 1.0 & 1.0 & 0.0 & 0.0 & 2.902 \\
\hline
\end{tabular}




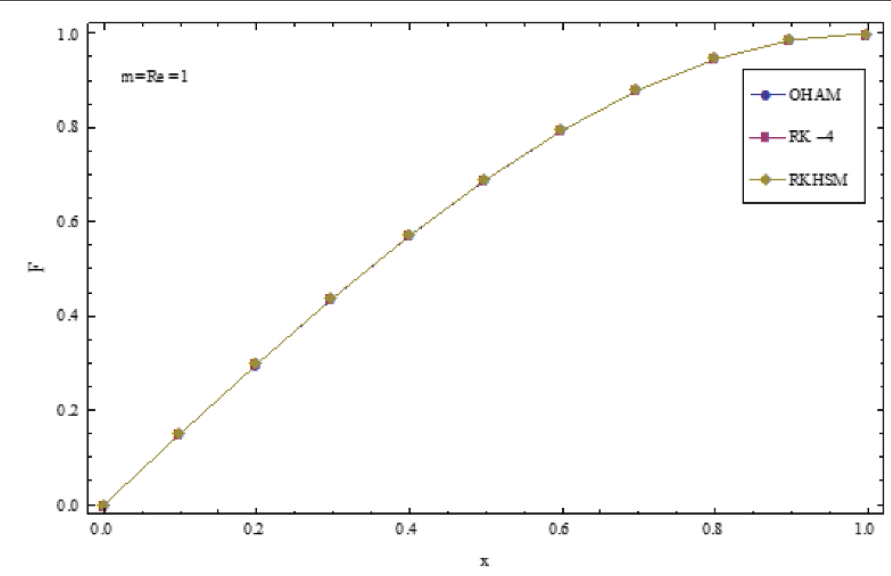

Figure 2 Comparison RKHSM, OHAM and RK-4 solutions for $m=\operatorname{Re}=1$.

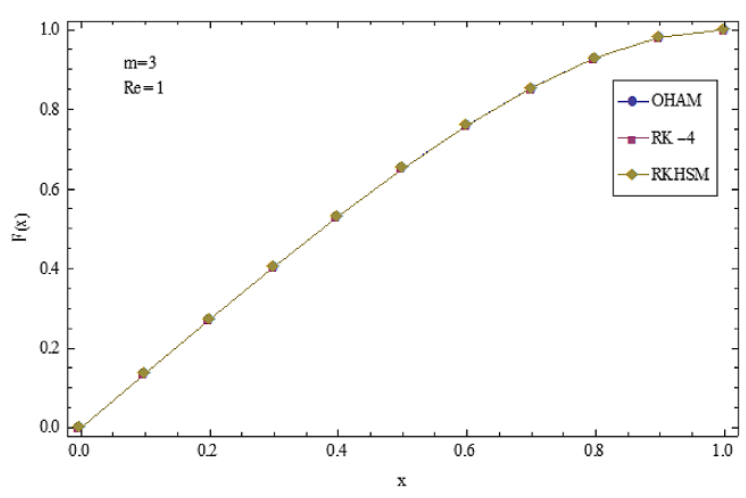

Figure 3 Comparison RKHSM, OHAM and RK-4 solutions for $m=3$ and $\mathrm{Re}=1$.

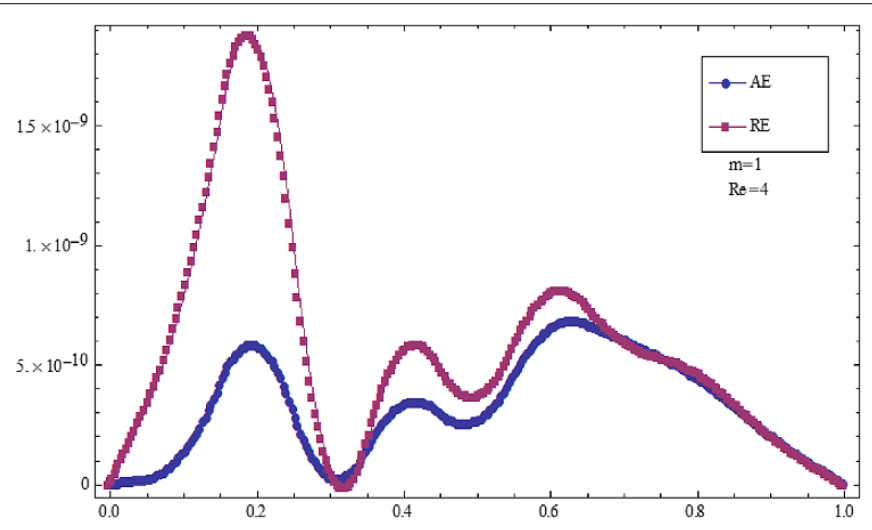

Figure $4 \mathrm{AE}$ and $\mathrm{RE}$ for $m=1$ and $\mathrm{Re}=4$. 

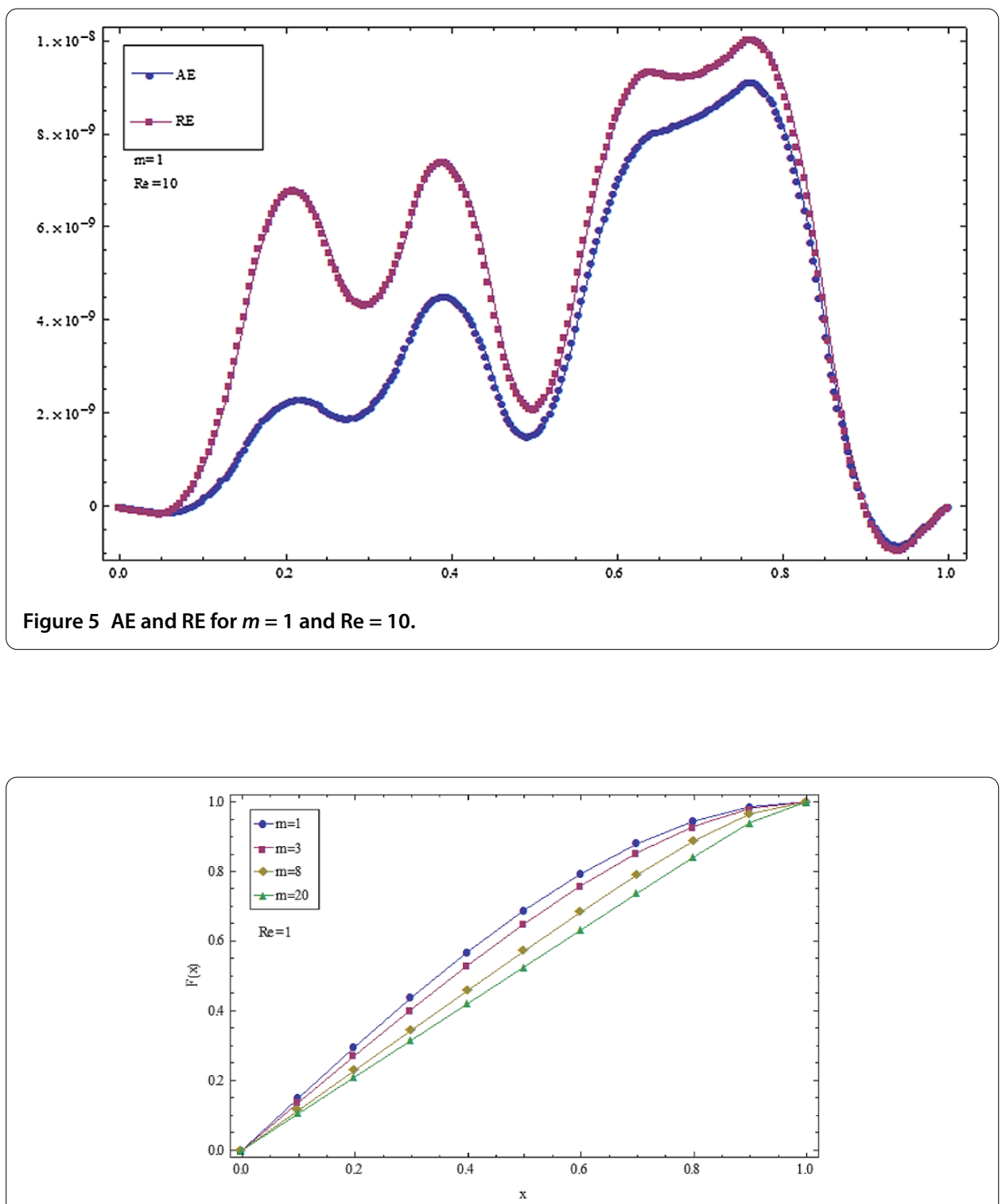

Figure 6 Comparison of squeezing flow for a fixed Reynolds number $\operatorname{Re}=1$ and increasing magnetic field effect $m=1,3,8,20$.

\section{Conclusion}

In this paper, we introduced an algorithm for solving the MHD squeezing fluid flow. We applied a new powerful method RKHSM to the reduced nonlinear boundary value problem. The approximate solution obtained by the present method is uniformly convergent. Clearly, the series solution methodology can be applied to much more complicated nonlinear differential equations and boundary value problems. However, if the problem becomes nonlinear, then the RKHSM does not require discretization or perturbation and it does not make closure approximation. Results of numerical examples show that the present method is an accurate and reliable analytical method for this problem. 


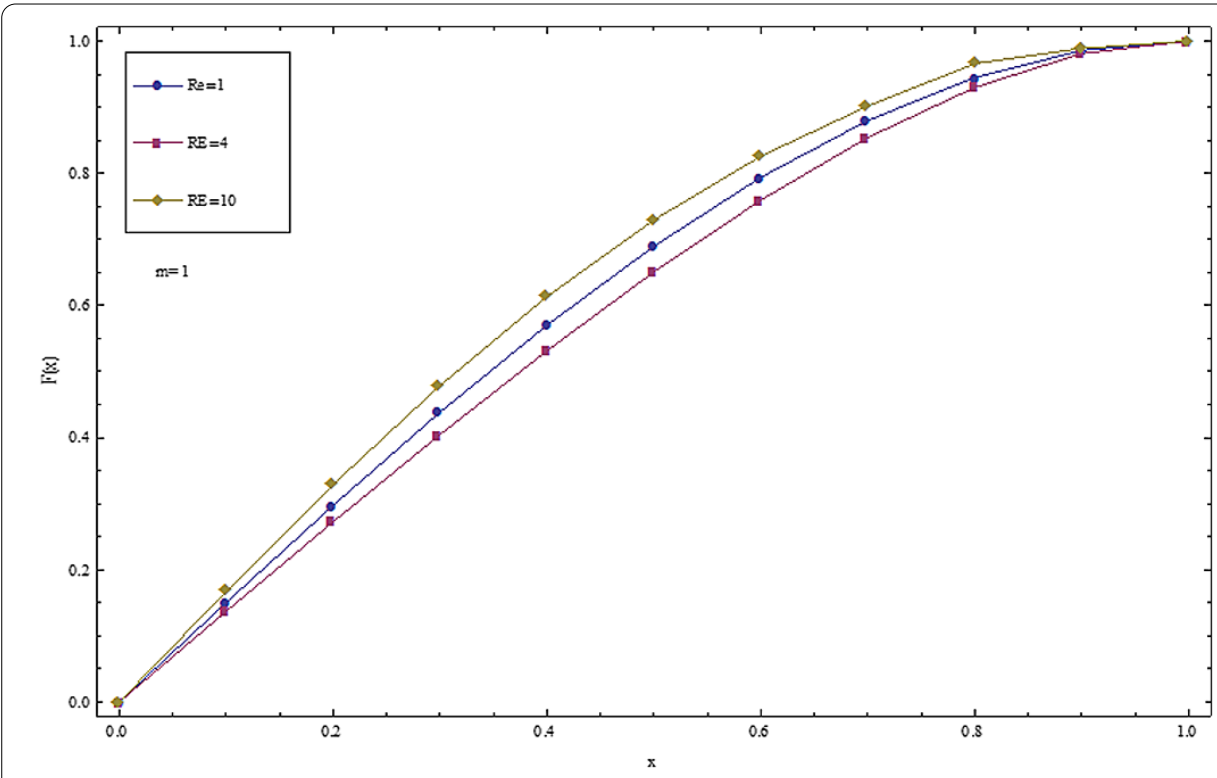

Figure 7 Comparison of squeezing flow for a fixed magnetic field effect $m=1$ and increasing Reynolds numbers $\operatorname{Re}=1,4,10$.

\section{Appendix}

Proof of Theorem 3.1 Let $u \in W_{2}^{5}[0,1]$. By Definition 3.2 we have

$$
\left\langle u, R_{y}\right\rangle_{W_{2}^{5}}=\sum_{i=0}^{4} u^{(i)}(0) R_{y}^{(i)}(0)+\int_{0}^{1} u^{(5)}(x) R_{y}^{(5)}(x) d x .
$$

Through several integrations by parts for (A.1), we have

$$
\begin{aligned}
\left\langle u, R_{y}\right\rangle_{W_{2}^{5}}= & \sum_{i=0}^{4} u^{(i)}(0)\left[R_{y}^{(i)}(0)-(-1)^{(4-i)} R_{y}^{(9-i)}(0)\right] \\
& +\sum_{i=0}^{4}(-1)^{(4-i)} u^{(i)}(1) R_{y}^{(9-i)}(1)-\int_{0}^{1} u(x) R_{y}^{(10)}(x) d x .
\end{aligned}
$$

Note the property of the reproducing kernel

$$
\left\langle u, R_{y}\right\rangle_{W_{2}^{5}}=u(y) .
$$

Now, if

$$
\left\{\begin{array}{l}
R_{y}^{\prime}(0)+R_{y}^{(8)}(0)=0, \\
R_{y}^{(3)}(0)+R_{y}^{(6)}(0)=0, \\
R_{y}^{(4)}(0)-R_{y}^{(5)}(0)=0, \\
R_{y}^{(5)}(1)=0 \\
R_{y}^{(6)}(1)=0, \\
R_{y}^{(7)}(1)=0,
\end{array}\right.
$$


then (A.2) implies that

$$
R_{y}^{(10)}(x)=-\delta(x-y)
$$

when $x \neq y$

$$
R_{y}^{(10)}(x)=0
$$

and therefore

$$
R_{y}(x)= \begin{cases}\sum_{i=1}^{10} c_{i}(y) x^{i-1}, & x \leq y \\ \sum_{i=1}^{10} d_{i}(y) x^{i-1}, & x>y .\end{cases}
$$

Since

$$
R_{y}^{(10)}(x)=\delta(x-y)
$$

we have

$$
R_{y^{+}}^{(k)}(y)=R_{y^{-}}^{(k)}(y), \quad k=0,1,2,3,4,5,6,7,8
$$

and

$$
R_{y^{+}}^{(9)}(y)-R_{y^{-}}^{(9)}(y)=-1 .
$$

Since $R_{y}(x) \in W_{2}^{5}[0,1]$, it follows that

$$
R_{y}(0)=0, \quad R_{y}(1)=0, \quad R_{y}^{\prime}(1)=0, \quad R_{y}^{\prime \prime}(0)=0 .
$$

From (A.3)-(A.6), the unknown coefficients $c_{i}(y)$ and $d_{i}(y)(i=1,2, \ldots, 12)$ can be obtained. This completes the proof.

\section{Competing interests}

The authors declare that they do not have any competing or conflict of interests.

\section{Authors' contributions}

Both authors contributed equally to this paper.

\section{Author details}

${ }^{1}$ Department of Mathematics, Science Faculty, Fırat University, Elazığ, 23119, Turkey. ${ }^{2}$ Department of Mathematics, Education Faculty, Dicle University, Diyarbakır, 21280, Turkey. ${ }^{3}$ Department of Mathematics, Texas A\&M

University-Kingsville, Kingsville, USA.

\section{Acknowledgements}

We presented this paper in the International Symposium on Biomathematics and Ecology Education Research in 2013. We would like to thank the organizers of this conference and the reviewers for their kind and helpful comments on this paper. Ali Akgül gratefully acknowledge that this paper was partially supported by the Dicle University and the Firat University. This paper is a part of PhD thesis of Ali Akgül. 


\section{References}

1. Papanastasiou, TC, Georgiou, GC, Alexandrou, AN: Viscous Fluid Flow. CRC Press, Boca Raton (1994)

2. Stefa Hughes, WF, Elco, RA: Magnetohydrodynamic lubrication flow between parallel rotating disks. J. Fluid Mech. 13, 21-32 (1962)

3. Ghori, QK, Ahmed, M, Siddiqui, AM: Application of homotopy perturbation method to squeezing flow of a Newtonian fluid. Int. J. Nonlinear Sci. Numer. Simul. 8, 179-184 (2007)

4. Ran, XJ, Zhu, QY, Li, Y: An explicit series solution of the squeezing flow between two infinite plates by means of the homotopy analysis method. Commun. Nonlinear Sci. Numer. Simul. 14, 119-132 (2009)

5. Grimm, RG: Squeezing flows of Newtonian liquid films an analysis include the fluid inertia. Appl. Sci. Res. 32, 149-166 (1976)

6. Kamiyama, S: Inertia effects in MHD hydrostatic thrust bearing. J. Lubr. Technol. 91, 589-596 (1969)

7. Hamza, EA: The magnetohydrodynamic squeeze film. J. Tribol. 110, 375-377 (1988)

8. Bhattacharya, S, Pal, A: Unsteady MHD squeezing flow between two parallel rotating discs. Mech. Res. Commun. 24, 615-623 (1997)

9. Geng, F, Cui, M: Solving a nonlinear system of second order boundary value problems. J. Math. Anal. Appl. 327, 1167-1181 (2007)

10. Zhou, Y, Lin, Y, Cui, M: An efficient computational method for second order boundary value problems of nonlinear differential equations. Appl. Math. Comput. 194, 357-365 (2007)

11. Yao, H, Cui, M: A new algorithm for a class of singular boundary value problems. Appl. Math. Comput. 186, 1183-1191 (2007)

12. Wang, W, Cui, M, Han, B: A new method for solving a class of singular two-point boundary value problems. Appl. Math. Comput. 206, 721-727 (2008)

13. Wang, YL, Chao, L: Using reproducing kernel for solving a class of partial differential equation with variable-coefficients. Appl. Math. Mech. 29, 129-137 (2008)

14. Li, F, Cui, M: A best approximation for the solution of one-dimensional variable-coefficient Burgers' equation. Numer. Methods Partial Differ. Equ. 25, 1353-1365 (2009)

15. Zhou, S, Cui, M: Approximate solution for a variable-coefficient semilinear heat equation with nonlocal boundary conditions. Int. J. Comput. Math. 86, 2248-2258 (2009)

16. $\mathrm{Du}, \mathrm{J}, \mathrm{Cui}, \mathrm{M}$ : Solving the forced Duffing equations with integral boundary conditions in the reproducing kernel space. Int. J. Comput. Math. 87, 2088-2100 (2010)

17. Lv, X, Cui, M: An efficient computational method for linear fifth-order two-point boundary value problems. J. Comput. Appl. Math. 234, 1551-1558 (2010)

18. Du, J, Cui, M: Constructive proof of existence for a class of fourth-order nonlinear BVPs. Comput. Math. Appl. 59, 903-911 (2010)

19. $\mathrm{Wu}, \mathrm{BY}, \mathrm{Li}, \mathrm{XY}$ : Iterative reproducing kernel method for nonlinear oscillator with discontinuity. Appl. Math. Lett. 23 1301-1304 (2010)

20. Cui, M, Lin, Y: Nonlinear Numerical Analysis in the Reproducing Kernel Spaces. Nova Science Publishers, New York (2009)

21. Lü, X, Cui, M: Analytic solutions to a class of nonlinear infinite-delay-differential equations. J. Math. Anal. Appl. 343 724-732 (2008)

22. Jiang, W, Cui, M: Constructive proof for existence of nonlinear two-point boundary value problems. Appl. Math. Comput. 215, 1937-1948 (2009)

23. Cui, M, Du, H: Representation of exact solution for the nonlinear Volterra-Fredholm integral equations. Appl. Math Comput. 182, 1795-1802 (2006)

24. Jiang, W, Lin, Y: Representation of exact solution for the time-fractional telegraph equation in the reproducing kernel space. Commun. Nonlinear Sci. Numer. Simul. 16, 3639-3645 (2011)

25. Lin, Y, Cui, M: A numerical solution to nonlinear multi-point boundary-value problems in the reproducing kernel space. Math. Methods Appl. Sci. 34, 44-47 (2011)

26. Mohammadi, M, Mokhtari, R: Solving the generalized regularized long wave equation on the basis of a reproducing kernel space. J. Comput. Appl. Math. 235, 4003-4014 (2011)

27. $\mathrm{Wu}, \mathrm{BY}, \mathrm{Li}, \mathrm{XY}$ : A new algorithm for a class of linear nonlocal boundary value problems based on the reproducing kernel method. Appl. Math. Lett. 24, 156-159 (2011)

28. Yao, H, Lin, Y: New algorithm for solving a nonlinear hyperbolic telegraph equation with an integral condition. Int. 」. Numer. Methods Biomed. Eng. 27, 1558-1568 (2011)

29. Inc, M, Akgül, A: The reproducing kernel Hilbert space method for solving Troesch's problem. J. Assoc. Arab Univ. Basic. Appl. Sci. 14, 19-27 (2013)

30. Inc, M, Akgül, A, Geng, F: Reproducing kernel Hilbert space method for solving Bratu's problem. Bul. Malays. Math. Sci. Soc. (in press)

31. Inc, M, Akgül, A, Kilicman, A: Explicit solution of telegraph equation based on reproducing kernel method. J. Funct. Spaces Appl. 2012, Article ID 984682 (2012)

32. Inc, M, Akgül, A, Kilicman, A: A new application of the reproducing kernel Hilbert space method to solve MHD Jeffery-Hamel flows problem in non-parallel walls. Abstr. Appl. Anal. 2013, Article ID 239454 (2013)

33. Inc, M, Akgül, A, Kilicman, A: On solving KdV equation using reproducing kernel Hilbert space method. Abstr. Appl. Anal. 2013, Article ID 578942 (2013)

34. Inc, M, Akgül, A, Kilicman, A: Numerical solutions of the second-order one-dimensional telegraph equation based on reproducing kernel Hilbert space method. Abstr. Appl. Anal. 2013, Article ID 768963 (2013)

35. Akram, G, Rehman, HU: Numerical solution of eighth order boundary value problems in reproducing Kernel space. Numer. Algorithms 62(3), 527-540 (2013)

36. Wenyan, $\mathrm{W}, \mathrm{Bo}, \mathrm{H}, \mathrm{Mas}$ ahiro, Y: Inverse heat problem of determining time-dependent source parameter in reproducing kernel space. Nonlinear Anal., Real World Appl. 14(1), 875-887 (2013)

37. Mokhtari, R, İsfahani, FT, Mohammadi, M: Reproducing kernel method for solving nonlinear differential-difference equations. Abstr. Appl. Anal. 2012, Article ID 514103 (2012) 
38. Islam, S, Ullah, M, Zaman, G, Idrees, M: Approximate solutions to MHD squeezing fluid flow. J. Appl. Math. Inform. 29(5-6), 1081-1096 (2011)

39. Idrees, M, Islam, S, Haq, S, Islam, S: Application of the optimal homotopy asymptotic method to squeezing flow. Comput. Math. Appl. 59, 3858-3866 (2010)

40. Mohyuddin, MR, Gotz, T: Resonance behavior of viscoelastic fluid in Poiseuille flow in the presence of a transversal magnetic field. Int. J. Numer. Methods Fluids 49, 837-847 (2005)

10.1186/1687-2770-2014-18

Cite this article as: Inc and Akgül: Approximate solutions for MHD squeezing fluid flow by a novel method. Boundary Value Problems 2014, 2014:18

Submit your manuscript to a SpringerOpen ${ }^{\circ}$ journal and benefit from:

- Convenient online submission

- Rigorous peer review

- Immediate publication on acceptance

Open access: articles freely available online

- High visibility within the field

- Retaining the copyright to your article

Submit your next manuscript at $>$ springeropen.com 